\title{
BLOCKCHAIN TECHNOLOGY IMPLEMENTATION ON MEDICAL RECORDS DATA MANAGEMENT: A REVIEW OF RECENT STUDIES
}

\author{
Dian Budi Santoso ${ }^{a^{*}}$; Anis Fuad ${ }^{b}$;Guntur Budi Herwanto ${ }^{c}$; Ahmad Watsiq Maula ${ }^{d}$ \\ $a^{*}$ Vocational College Universitas Gadjah Mada ; Sekip Unit 1 ; \\ Yogyakarta 55281 ; Indonesia \\ ${ }^{b, d}$ Faculty of Medicine ; Public Health and Nursing Universitas Gadjah Mada ; Sekip \\ Utara ; Yogyakarta 55281 ; Indonesia \\ ${ }^{c}$ Faculty of Mathematics and Natural Science Universitas Gadjah Mada ; Sekip Utara ; \\ Yogyakarta 55281 ; Indonesia
}

\begin{abstract}
Blockchain first introduced and implemented in digital currency management and transactions. Its application to medical records data management is a novelty. This paper described the implementation of blockchain technology in the health care industry, especially in medical records data management A literature review was conducted on three popular databases, ScienceDirect, SpringerLink, and IEEE Xplore with the keywords "health", "medical record" and "blockchain" with "research article" and "conference proceeding" filters. There are a few articles that meet the criteria to review indicated that the implementation of blockchain technology in medical records data management is a novelty and still in the early phase. Blockchain is a potential technology in supporting the implementation of electronic medical records, especially related to data integration and privacy. Several scientific publications related to the implementation of blockchain for medical records data management shown that the implementation of this technology will make the patient have full control over their health data. Yet there are still many challenges in the implementation both from the user side and the technology infrastructure.
\end{abstract}

Keywords: blockchain; electronic medical record; data integration

\section{Introduction}

Indonesian health care facilities are beginning to shift from paper-based medical records to an electronic one. An electronic medical records is a computerized health information system that stores detailed patient demographic data, medical history, allergy records, laboratory examination results and some are equipped with decision support systems (Ludwick \& Doucete, 2009).

Although it has begun to switch to electronic records, each health care facility builds its own system and not integrated with other health care facilities. Communication or data exchange among health care facilities becomes an important key to obtain

\footnotetext{
*) Corresponding Author (Dian Budi Santoso)

E-mail: dianbudisantoso@ugm.ac.id
}

comprehensive patient data. Associated with patient data exchange, the implementation of electronic medical records also raises new issues related to data security and privacy (Keshta \& Odeh, 2020).

The issues, challenges, and problems faced in the implementation of electronic medical records could be overcome with blockchain technology that's known as the main technology of bitcoin digital currency (Heilman, Baldimtsi, \& Goldberg, 2016). Blockchain is a distributed database system that can track and browse any data stored on it. Data transaction in the blockchain is sorted by the blocks. Each block consists of data, a time transaction record, and a link to the other block containing previous data transactions. Each block has a digital identity that will be encrypted with certain algorithms 
that makes it difficult to hack. The blocks are stored on each node in the network in a decentralized fashion that makes the data irreversible and unchangeable (Swan, 2015). Block's data can only be added and cannot be changed because changing the data on one block will affect the other blocks so it will be rejected by the blockchain system. This technology concept will ensure that data in blockchain system are safe from hackers who want to change the data.

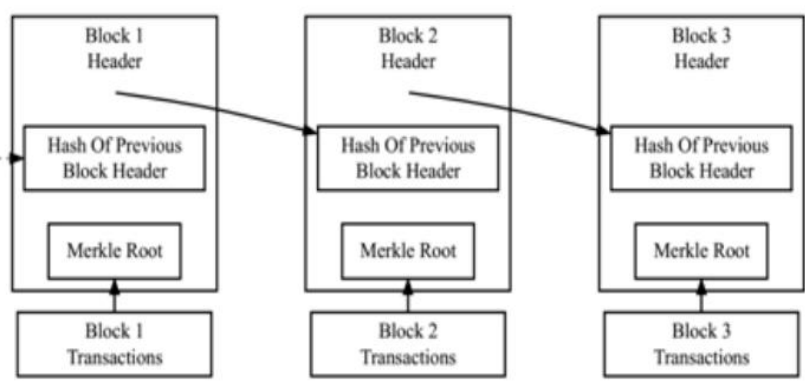

Figure 1. Blocks connections on blockchain (Seitz \& Wickramasinghe, 2017)

Blockchain data are stored in different places that connected through a peer to peer network make it immune from damage and loss (Decker \& Wattenhofer, 2013). Unlike a centralized database system where data on the server is lost then all clients will also lose the data. In blockchain, data can easily be traced and verified by comparing the data blocks stored in each node in the network. If there is a new data block formed, the node that forms the new data block will broadcast on all nodes connected in the network. The new data block will be synchronized to all nodes in blockchain system that makes each node store the information of the entire data block.

With the blockchain implementation, each patient's medical records will be well maintained and secure. In addition, patients can arrange themselves to whom their health information can be shared. The more health care facilities connected to the blockchain system, the more complete and comprehensive the patient data. This article was written to explain the implementation of blockchain technology in the health care industry, especially in health data management based on recent studies that have been published.

\section{Method}

A literature review was conducted to find out the implementation of blockchain technology in the health care industry, especially in medical records data management. Scientific publications covering the implementation of blockchain in electronic medical records are sought in 3 popular scientific publication databases, ScienceDirect, SpringerLink, and IEEE Xplore with "medical record, "health" and "blockchain" keywords with "research article" and "conference proceeding" filters.

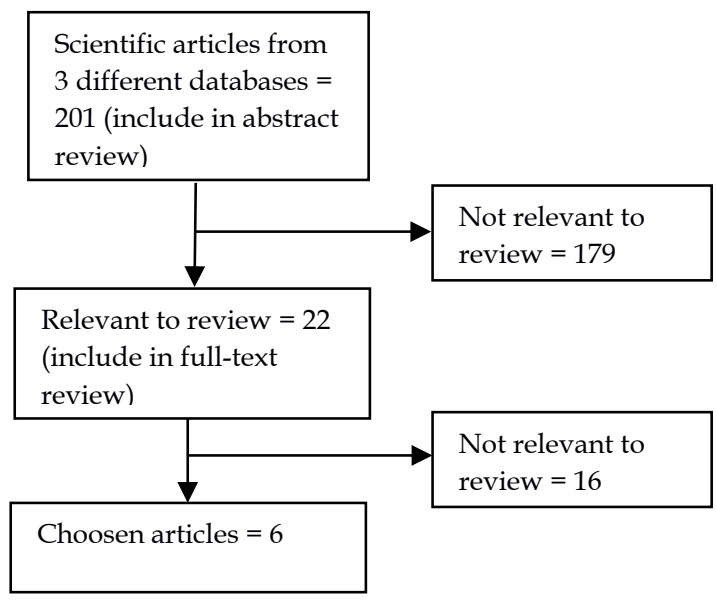

Figure 2. Selection process

201 articles from scientific journals and proceedings were found from 3 databases. By abstract review, 22 articles relevant to the topic to be discussed were chosen to include in fulltext review. From this 22 article, based on fulltext review only 6 articles were chosen to be reviewed in this paper.

\section{Result and Discussion}

There are several scientific publications that discussed blockchain technology implementation on medical records data management. In general, the implementation of this technology is related to the data inteoperability data between health facilities and related to the patient's right to be able to share or not his health record and to whomever the data will be shared. There 6 published study about product or prototype that using blockchain to manage the medical records data. 
Tabel 1. Published blockchain based product or prototype to manage medical records data by years

\begin{tabular}{|c|c|c|}
\hline Year & $\begin{array}{c}\text { Number of a } \\
\text { published product }\end{array}$ & Product's name \\
\hline 2019 & 2 & $\begin{array}{l}\text { MedBloc (Huang, et } \\
\text { al., 2019), } \\
\text { MedChain } \\
\text { (Daraghmi, et al., } \\
\text { 2019) }\end{array}$ \\
\hline 2018 & 1 & $\begin{array}{c}\text { Ancile (Dagher et } \\
\text { al., 2018) }\end{array}$ \\
\hline 2017 & 1 & $\begin{array}{l}\text { Omni PHR (Roehrs } \\
\text { et al., 2017) }\end{array}$ \\
\hline 2016 & 2 & $\begin{array}{l}\text { health care Data } \\
\text { Gateway (Yue et al., } \\
\text { 2016)., MedRec } \\
\text { (Azaria et al., 2016) }\end{array}$ \\
\hline
\end{tabular}

\section{MedRec}

MedRec is an electronic medical records with the concept of distributed data storage using blockchain technology (Azaria et al., 2016). For example, if patient $A$ went to hospital $B$ then the data will be stored in hospital $B$, then if he went to hospital $C$ then the data will be stored in hospital C. With patient approval through an application interface, the patient data at hospital $\mathrm{B}$ is also accessible to hospital C. The patient's medical history can be traced completely until the last health care episode with certain rules agreed in blockchain system.

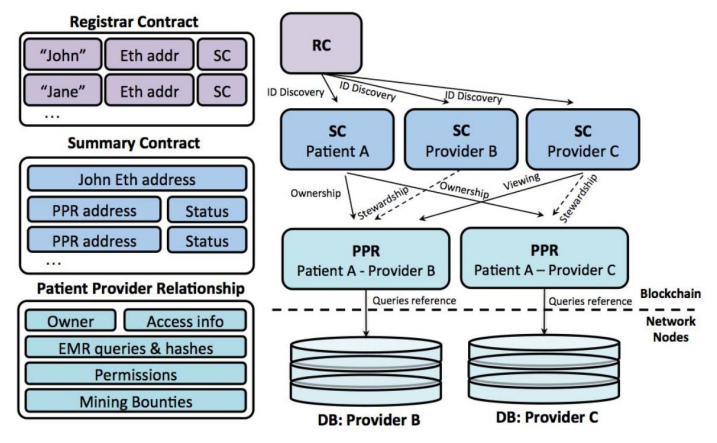

Figure 3. MedRec integrate electronic medical records data between health care facilities using blockchain system (Azaria et al., 2016)

\section{health care Data Gateway}

health care Data Gateway is a mobile application with blockchain-based architecture that enables patients to safely and conveniently store, control and share their medical data without worrying about their privacy (Yue et al.,
2016). The user or patient will receive a notification if there is a request for their medical data. The patient can choose which data will be sent to the requester through a mobile application interface.
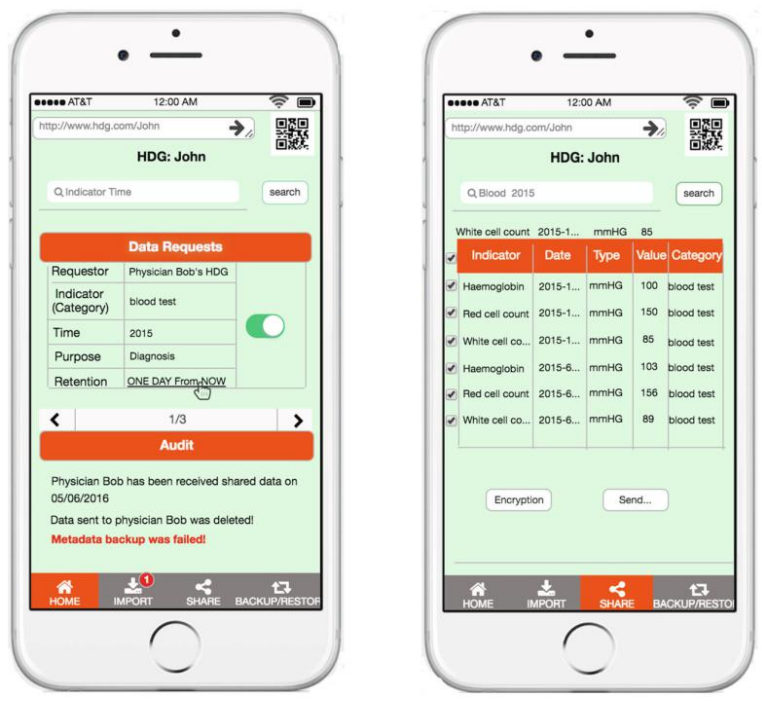

Figure 4. Data request and transmission through health care Data Gateway (Yue et al., 2016)

\section{OMniPHR}

OmniPHR is a distributed personal health record model that linked in a peer to peer network system (Roehrs et al., 2017). The patient's health record data are stored in different places but can be accessed with the patient's permission. The health records data could come from patients themselves such as health mobile apps and wearable devices, and also come from hospitals, doctors, and clinical laboratories.

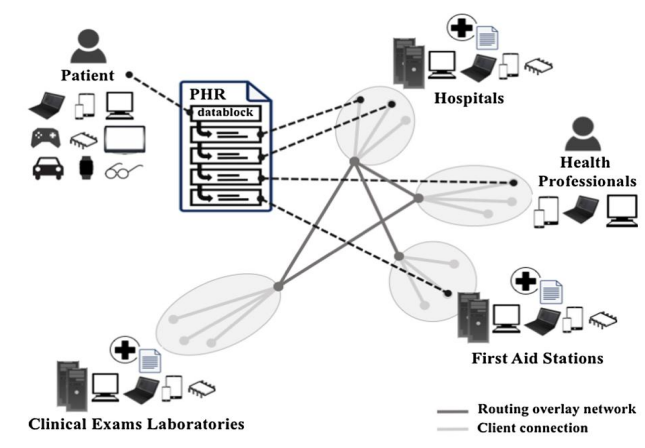

Figure 5. OmniPHR data distributed through peer to peer network (Roehrs et al., 2017) 


\section{Ancile}

Ancile is a blockchain-based framework for the security, interoperability, and efficiency of access towards medical records from patients, health facilities and third parties such as health insurance providers for example by maintaining the patient's privacy aspects of sensitive health information (Dagher et al., 2018). Patients, health facilities, and third parties can interact safely through Ancile.

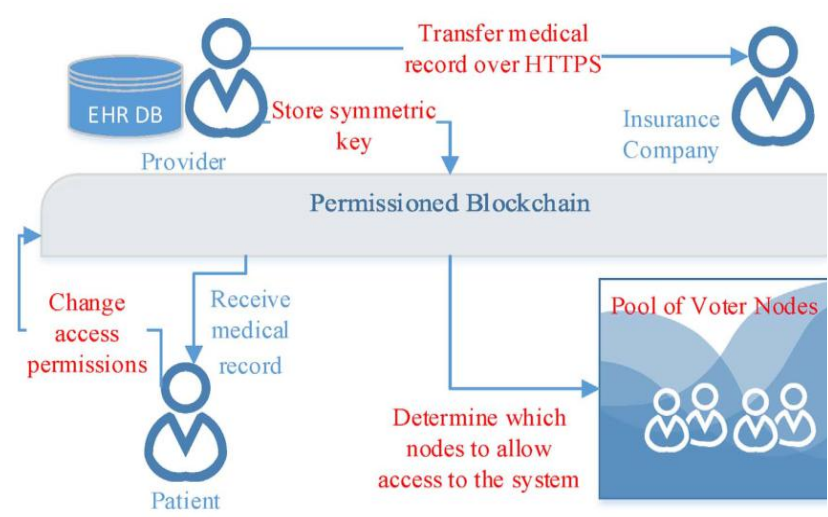

Figure 6. Ancile capabilities to interact users with the blockchain system (Dagher et al., 2018)

\section{MedChain}

MedChain, like Ancile, designed to provide secure, interoperable and effective access to medical records data by health care providers and patients while keeps the privacy of the patients (Daraghmi, et al., 2019). The difference is MedChain has incentive mechanism. Based on several blockchain protocols, an incentive will be given as a reward or transaction fee for mining block. The MedRec gave incentives to health providers for their participation in mining to earn an Ether, which is an Ethereuem based currency unit. Since the majority of the current health care systems are not intended to involve any monetary value for medical records data, and there is an issue about completeness and quality of medical records data there is a need to add an incentive mechanism for the electronic medical records systems.

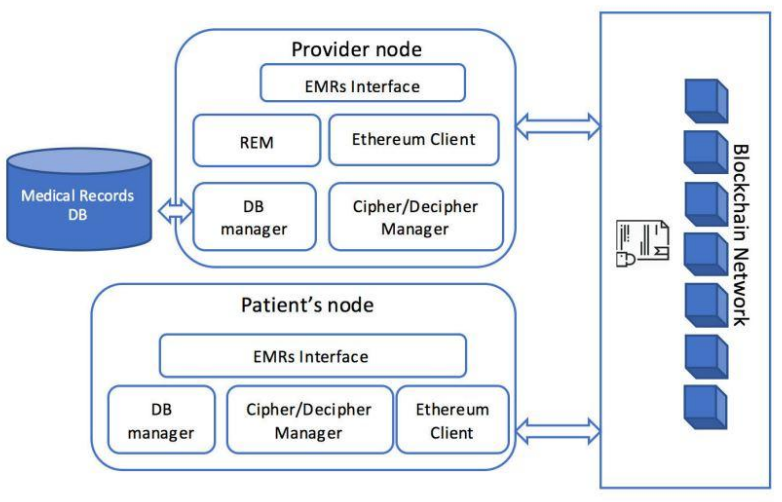

Figure 7. Software components of the MedChain system (Daraghmi, et al., 2019)

\section{MedBloc}

MedBloc was present to demonstrate how the blockchain can establish New Zealand's first shared electronic health records (Huang, et al., 2019). Using MedBloc, health care organizations, such as hospitals, medical centers, and Specialists can integrate their data through blockchain technology. MedBlock showed that blockchain has the potential to disrupt the entire health technology sector.

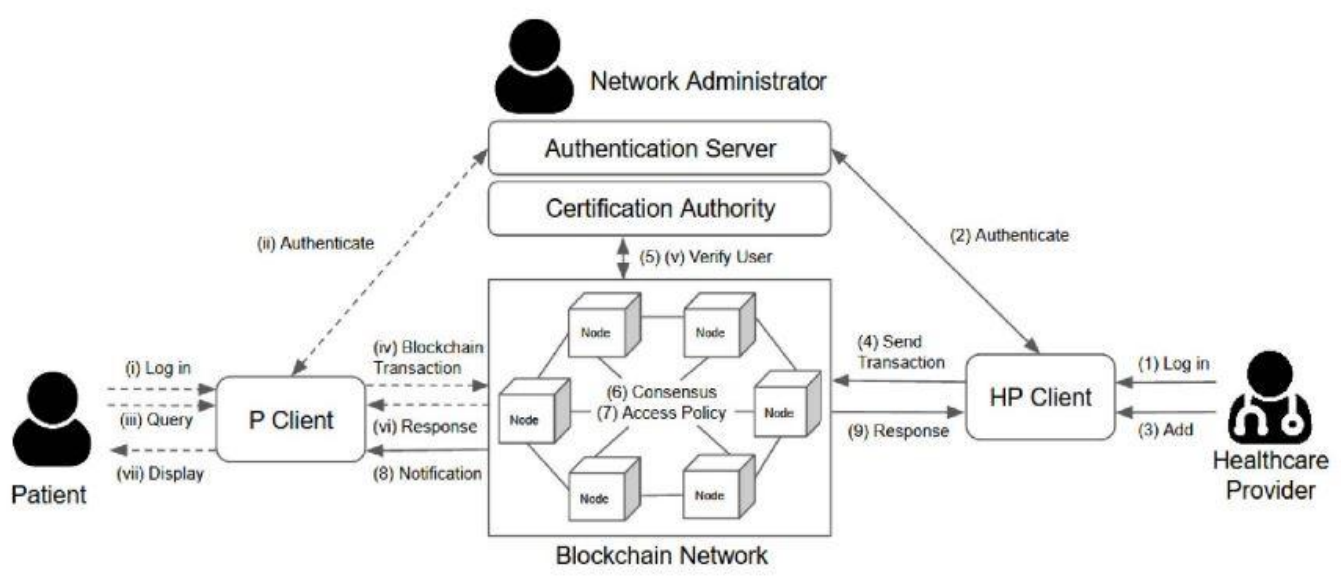

Figure 8. The MedBloc Architecture (Huang, et al., 2019). 
A "P Client" in Figure 8 is patients' client service, and an "HP Client" is health care providers' client service. The Blockchain Network consists of several nodes. Solid lines with arrows represent a system process when the health care provider tries to add a new record for the patient. Dotted lines with arrows represent a system process when a patient tries to see their health record.

Only a few relevant articles are available for review in this study indicating that the implementation of blockchain technology in medical records data management is a novelty. There is no publication about blockchain implementation on medical records data management below 2016. Until 2015, we only recognize blockchain technology implemented in digital currency transactions such as bitcoin which is currently on the rise (Godsiff, 2015). Its implementation in medical records data management will be a new breakthrough that can meet the challenges of implementing electronic medical records related to data security and privacy. The patient can control whomever the data or medical records will be shared. Of course, this concept should also be well-formulated especially in emergencies condition where the patient cannot access the system because of their physical conditions.

Blockchain uses distributed databases concept, data stored on various storage areas connected to a blockchain network system. This capability makes blockchain could overcome data disintegration that becomes one of the problems of electronic medical records implementation. Blockchain also makes electronic medical records become more interoperable. As with the MedRec concept, although patients have been treated in different hospitals and the data also stored on the local storage of the hospital, by using blockchain technology the data can be integrated (Azaria et al., 2016). The patient's medical history can be obtained intact in every node in the blockchain network system.

Blockchain also allows the patient data to be provided anonymously by organizing what data is encrypted and not encrypted. This will be particularly applicable in the provision of data for research interests as well as government health-related policy making. Data is provided anonymously so that the privacy of each individual patient will be maintained. The government and researchers can access the required data easily. The more electronic medical records systems connected in the blockchain network the more data available to access and analyze.

With many advantages of course blockchain technology become a very potential platform to support the implementation of electronic medical records while maintaining patient privacy. Nevertheless, there are still many challenges to implement blockchain for medical records data management. There are still many people who have not considered health data as valuable data, while the implementation of blockchain in electronic medical records requires the active role of data owners. In addition to preparing the technology, educating patients or potential users are required to make them play an active role independently. Incentive mechanism like in MedChain also worth to adopt.

In addition, due to its distributed nature, blockchain required large data storage which has implications for the prepared cost (Wood, 2014). This makes the blockchain system inefficient for storing large size data such as $\mathrm{x}$ ray images. Blockchain can be used to control who can access the data, but the data itself must be stored in multiple locations in a distributed manner that could have a weak security system that can be attacked from the outside blockchain system. Each node connected to the blockchain network must have an adequate security system to anticipate if anyone is attempting to steal the data directly on its local storage. A study and discussion about risks management and a standardize framework for the sustainable implementation of blockchain has not yet emerge (Drljevic et al., 2020), especially in heath sector.

\section{Conclusion and Suggestion}

Blockchain is a potential technology in supporting the implementation of electronic medical records, especially related to data integration and privacy. Several scientific papers related to the implementation of blockchain in medical records data management indicate that the implementation of this technology will make the patient have full control over their health data. Nevertheless, there are still many challenges in the implementation both from the user side and the technology infrastructure, further studies are needed in this regard. 


\section{Acknowledgments}

Thanks to Universitas Gadjah Mada for facilitating free access to scientific journals which are used as material and references in this study.

\section{References}

Azaria, A., Ekblaw, A., Halamka, J. D., \& Lippman, A. (2016). MedRec: Using Blockchain for Medical Data Access and Permission Management. 2nd International Conference on Open \& Big Data. IEEE.

Dagher, G. G., Mohler, J., Milojkovic, M., Marella, P. B. (2018). Ancile: Privacypreserving framework for access control and interoperability of electronic health records using blockchain technology. Sustainable Cities and Society, 39, 283297.

Daraghmi, E., Daraghmi, Y., Yuan, S. (2019). MedChain: A Design of BlockchainBased System for Medical Records Access and Permissions Management. IEEE Access, 7, 164595-164613.

Decker, C., Wattenhofer, R. (2013). Information propagation in the Bitcoin network. IEEE Thirteenth International Conference on Peer-to-Peer Computing. Trento: IEEE.

Drljevic, N., Aranda, D. A., Stantchev, V. (2020). Perspectives on risks and standards that affect the requirements engineering of blockchain technology. Computer Standards \& Interfaces, 69, 1-7.

Godsiff, P. (2015). Bitcoin: Bubble or Blockchain. Smart Innovation, Systems and Technologies (pp. 191-203). Basel: Springer International Publishing AG.

Huang, J., Qi, Y., Asghar, M. R., Meads, A., Tu, Y. (2019). MedBloc: A Blockchain-based Secure EHR System for Sharing and Accessing Medical Data. 18th IEEE International Conference on Trust,
Security and Privacy in Computing and Communications, 594-601.

Heilman, E., Baldimtsi, F., \& Goldberg, S. (2016). Blindly Signed Contracts: Anonymous On-Blockchain and Off-Blockchain Bitcoin Transactions. International Conference on Financial Cryptography and Data Security (pp. 43-60). Basel: Springer International Publishing AG.

Keshta, I., Odeh, A. (2020). Security and privacy of electronic health records: Concerns and challenges. Egyptian Informatics Journal, 1-7.

Ludwick, D. A., \& Doucette, J. (2009). Adopting Electronic Medical Records in Primary, Lesson Learned from Health Information System Implementation Experience in Seven Countries. International Journal of Biomedical Informatics, 22-31.

Roehrs, A., da Costa, C. A., da Rosa Righi, R. (2017). OmniPHR: A distributed architecture model to integrate personal health records. Journal of Biomedical Informatics, 71, 70-81.

Swan, M. (2015). Blockchain: Blueprint for a New Economy. Sebastopol: O'Reilly Media Inc.

Seitz, J., \& Wickramasinghe, N. (2017). Blockchain technology in e-health: The case of electronic prescriptions in Germany. XVII International Scientific Conference on Industrial Systems (pp. 156-159). Novi Sad: University of Novi Sad.

Wood, G. (2014). Ethereum: A secure decentralized generalized transaction ledger. Accessed from http://gavwood.com/paper.pdf [March 31, 2020]

Yue, X., Wang, H., Jin, D., Li, M., \& Jiang, W. (2016). health care Data Gateways: Found health care Intelligence on Blockchain with Novel Privacy Risk Control. Journal of Medical Systems, 40. 